\title{
Cost-effectiveness analysis of everolimus plus exemestane versus exemestane alone for treatment of hormone receptor positive metastatic breast cancer
}

\author{
Vakaramoko Diaby \\ Division of Economic, Social and Administrative Pharmacy, College of Pharmacy and \\ Pharmaceutical Sciences, Florida A\&M University (FAMU), Tallahassee, FL, USA \\ Georges Adunlin \\ Division of Economic, Social and Administrative Pharmacy, College of Pharmacy and \\ Pharmaceutical Sciences, Florida A\&M University (FAMU), Tallahassee, FL, USA
}

\section{Simon B. Zeichner}

Department of Internal Medicine, Mount Sinai Medical Center, Miami Beach, FL, USA

Kiran Avancha

Hartford Healthcare Cancer Institute, Hartford, CT, USA

\section{Gilberto Lopes}

HCor Onco, São Paulo, Brazil

Centro Paulista de Oncologia, São Paulo, Brazil

Johns Hopkins University, Baltimore, MD, USA

\section{Stefan Gluck}

Division of Hematology/Oncology, Department of Medicine, Sylvester Comprehensive Cancer

Center, Leonard M. Miller School of Medicine, University of Miami, Coral Gables, FL, USA

\author{
Alberto J. Montero \\ Department of Solid Tumor Oncology, Taussig Cancer Center, Cleveland, OH, USA
}

\begin{abstract}
Everolimus in combination with exemestane significantly improved progression-free survival compared to exemestane alone in patients previously treated with non-steroidal aromatase inhibitors in the BOLERO-2 trial. As a result, this combination has been approved by the food and drug administration to treat postmenopausal women with hormone receptor positive and HER2 negative metastatic breast cancer. A cost-effectiveness analysis was conducted to determine whether everolimus represents good value for money, utilizing data from BOLERO-2. A decisionanalytic model was used to estimate the incremental cost-effectiveness ratio between treatment arms of the BOLERO-2 trial. Costs were obtained from the Center for Medicare Services drug
\end{abstract}

\footnotetext{
(C) Springer Science+Business Media New York 2014 diaby_v@yahoo.fr.

Conflict of interest The authors certify that they have no conflict of interest with any financial organization regarding the material discussed in the manuscript.
} 
payment table and physician fee schedule. Benefits were expressed as quality-adjusted progression-free survival weeks (QAPFW) and quality-adjusted progression-free years (QAPFY), with utilities/disutilities derived from the literature. Deterministic and probabilistic sensitivity analyses were performed. A willingness to pay threshold of 1-3 times the per capita gross domestic product was adopted, as per the definition of the World Health Organization. The U.S. per capita gross domestic product in 2013 was $\$ 49,965$; thus, a threshold varying between $\$ 49,965$ and $\$ 149,895$ was considered. Everolimus/exemestane had an incremental benefit of 11.88 QAPFW (0.22 QAPFY) compared to exemestane and an incremental cost of $\$ 60,574$. This translated into an ICER of $\$ 265,498.5 / \mathrm{QAPFY}$. Univariate sensitivity analyses showed important variations of the ICER, ranging between $\$ 189,836.4$ and \$530,947/QAPFY. A tornado analysis suggested that the key drivers of our model, by order of importance, included health utility value for stable disease, everolimus acquisition costs, and transition probabilities from the stable to the progression states. The Monte-Carlo simulation showed results that were similar to the base-case analysis. This cost-effectiveness analysis showed that everolimus plus exemestane is not costeffective compared to exemestane alone. Further research is needed to investigate the costeffectiveness of the drug combination within sub-groups of the population studied in BOLERO-2.

\section{Keywords}

Metastatic breast cancer; Aromatase inhibitor therapy; Everolimus; Exemestane; Health-related quality of life; Progression-free survival; BOLERO-2; Cost-effectiveness; Analysis

\section{Introduction}

Excluding cancers of the skin, breast cancer is the most frequently diagnosed cancer in women [1]. In the United States (U.S.), an estimated 232,340 new cases of invasive breast cancer were diagnosed among women in 2013 [1]. Breast cancer ranks second as a cause of cancer death in women (after lung cancer), with 39,620 women expected to die from breast cancer in 2013 [1].

Endocrine therapy remains the gold standard for the treatment of hormone receptor (HR) positive metastatic breast cancer $(\mathrm{mBC})$. Due to increased efficacy and a favorable toxicity profile, endocrine therapy with either aromatase inhibitors (AI) or fulvestrant remains the preferred first-line treatment for postmenopausal women with either HR positive or HER2 negative $\mathrm{mBC}$. Unfortunately, endocrine therapy resistance invariably develops in most patients and therefore still remains a major clinical problem in the management of HR positive metastatic breast cancer. Acquired endocrine resistance occurs as the result of complex adaptive changes in breast cancer cells that come from the long-term exposure to endocrine treatment. A better understanding of the biologic drivers of endocrine resistance in breast cancer has led to the development of novel targeted therapies that can be combined with AI to improve their overall effectiveness [2].

Preclinical studies have shown that the phosphatidylinositol 3-kinase (PI3K)-Aktmammalian target of the rapamycin (mTOR) signaling pathway is important to endocrine resistance [3, 4]. In preclinical models, combining everolimus (Afinitor ${ }^{\circledR}$ ), an mTOR inhibitor, with an AI produced synergistic anti-tumor activity [5]. Subsequently, data from a 
phase 2 randomized trial [6] and from the more recent phase 3 BOLERO-2 trial [7] demonstrated that everolimus in combination with an AI significantly prolonged progression-free survival (PFS) compared with AI therapy alone in women with hormone receptor positive MBC. Following the interim results of the BOLERO-2 RCT in 2012 [7], everolimus was approved for use in combination with exemestane (an AI) by the European Medicines Agency (EMA) and the U.S. Food and Drug Administration (FDA). The combination is indicated for postmenopausal women with hormone receptor positive, HER2 negative $\mathrm{mBC}$ with recurrence, or progression of their cancer after treatment with letrozole or anastrozole [7]. Mature overall survival data from the BOLERO-2 trial have not yet been published; however, recent data reported in abstract form suggest that there was no significant difference in OS between the groups. The EMA and FDA commonly approve drugs for the treatment of breast cancer based on PFS alone. Therefore, it is important for cost-effective analyses to reflect this clinical reality. The combination of everolimus plus exemestane is now part of routine clinical practice, and a robust cost-effective analysis is needed in order to help payers make informed reimbursement decisions regarding this FDAapproved combination. The objective of this study was to determine the cost-effectiveness of everolimus plus exemestane compared to exemestane alone in postmenopausal women with HR positive $\mathrm{mBC}$, utilizing the published data from the BOLERO-2 trial.

\section{Materials and methods}

\section{Model overview}

A Markov model was developed to simulate the incremental cost-effectiveness ratio (ICER) of everolimus in combination with exemestane compared to exemestane alone. The population modeled was a hypothetical cohort of patients with HR positive advanced breast cancer who showed the same characteristics as those enrolled in the BOLERO-2 trial [7]. Analyses were performed using the decision-analytic software, TreeAge Pro 2013 (TreeAge Software Inc., Williamstown, MA, USA).

Three health states were considered in the Markov model: stable (progression-free) without adverse events, stable with adverse events, and progression. The health states were mutually exclusive, i.e., a patient could only experience a single health state at any given time. The model reflected a time horizon equivalent to that of the final progression-free analysis of the BOLERO-2 trial [8], i.e., 120 weeks. The cycle length was set at 6 weeks in order to match the patient assessment time interval used in the trial.

Figure 1 depicts how HR positive $\mathrm{mBC}$ patients travelled through the model. Patients entered the model at the point at which they would be considered eligible for endocrine therapy and received oral everolimus (10 mg daily), in conjunction with exemestane ( $25 \mathrm{mg}$ daily) or exemestane alone ( $25 \mathrm{mg}$ daily). At that point, all patients were in the stable with no adverse events state and would transition to the other health states based on transition probabilities. Patients who experience disease progression would exit the model. Hypothetical patients accrued costs and utilities through each cycle. Our model assumed that the occurrence of adverse events (AEs) would not affect the probability of progressing but would affect patient quality of life (utility decrements) and healthcare costs (increase). 
The analysis adopted a US healthcare government-run payer perspective (Medicare). Based on this perspective, the model included only direct medical costs. We used the definition for willingness to pay (WTP) threshold of 1-3 times the per capita gross domestic product (GDP) suggested by the World Health Organization (WHO). The U.S. per capita GDP in $2012 / 2013$ was $\$ 49,965$; thus, a WTP threshold varying between $\$ 49,965$ and $\$ 149,895$ U.S. dollars was considered.

\section{Model input parameters}

Transition probabilities between health states-Using the final PFS Kaplan-Meier curves of the BOLERO-2 trial, survival modeling techniques were applied to determine transition probabilities from stable (with or without adverse events) to progression-free states [9]. This application consisted in (i) reconstructing individual patient data (IPD) based on published final PFS Kaplan-Meier curves of the BOLERO-2 trial, (ii) fitting parametric distributions to reconstructed data, and (iii) estimating transition probabilities for Markov modeling. The log-logistic distribution most accurately represented the data. The formula for transition probabilities for treatment arm 0 (exemestane alone) and 1 (everolimus plus exemestane) was represented by Eqs. 1 and 2, respectively [9].

$$
\begin{aligned}
& t_{p}\left(t_{u}\right)=1-\exp \left\{[0.068025 \times(t-u)]^{\left(\frac{1}{0.5583247}\right)}-(0.068025 t)^{\left(\frac{1}{0.5583247}\right)}\right\}, \\
& t_{p}\left(t_{u}\right)=1-\exp \left\{[0.030142 \times(t-u)]^{\left(\frac{1}{0.6187177}\right)}-(0.030142 t)^{\left(\frac{1}{0.6187177}\right)}\right\},
\end{aligned}
$$

where $t_{p}$ represents the transition probability, $t_{u}$ the cycle for which the transition probability is estimated, $t-u$ representing previous stage (previous cycle), and $t$ representing the current stage (current cycle). The value 0.068025 is the scale of the distribution for treatment arm 0 $\left(\lambda_{0}\right)$. The value 0.5583247 is the shape of the distribution for treatment arm $0\left(\gamma_{0}\right)$. The value 0.030142 is the scale of the distribution for treatment arm $1\left(\lambda_{1}\right)$. The value 0.6187177 is the shape of the distribution for treatment arm $0\left(\gamma_{1}\right)$ [9]. More details can be obtained from the published tutorial [9]. Transition probabilities from stable without adverse events to stable with adverse events states were obtained directly from the BOLERO-2 trial [7]. The authors of the trial reported a 120-week probability of developing AEs of 0.11 for treatment arm 1 and of 0.01 for treatment arm 0 . By assuming that probabilities remained constant over time, they could be converted back into 6-week probabilities to match the cycle length of our Markov model.

Medical resource use and costs-Direct medical costs considered in this study were physician visits, acquisition costs of drugs, laboratory tests, computed tomography (CT) scans, and treatment for the most common grade 3 or 4 adverse events (Table 1). The majority of cost data were obtained from the Center for Medicare Services Drug Payment Table and Physician Fee Schedule and was represented in 2013 US dollars.

Costs and resource use associated with AEs were based on published literature [10]. The cost of the management of AEs was estimated based on the distribution of the most common 
grade 3-4 AEs reported in the BOLERO-2 trial (weighted average of common grade 3-4 AEs-see Table 2) and the published literature. This cost was only applied to the first cycle of entering stable disease with AEs. For the subsequent cycles, the costs of stable disease with AEs were considered identical to those of "stable disease without AEs".

Health utilities - Health utilities and disutilities (associated with common grade 3-4 AEs) were obtained from the published literature (see Table 2). Like the costs of the management of AEs, the disutility associated with AEs was only applied to the first cycle of entering stable disease with AEs. For the remaining cycles, the utility of stable disease with AEs was considered identical to that of stable disease without AEs.

Discounting and half-cycle correction-All costs incurred and utilities estimated during our analysis were discounted at a $3.5 \%$ annual rate [11, 12]. This annual discount rate was converted into a 6 -week discount using the formula below:

$$
r^{*}=(1+r)\left(\left(\frac{1}{w}\right) \times 6\right)-1,
$$

where $r^{*}$ is the 6-week discount rate, $r$ is the annual discount rate, and $\mathrm{w}$ is the total number of weeks composing a year (52). The "if function" in TreeAge was used to apply the 6-week discount rate to the cycles composing the temporal framework of our model. A variable "discount" was created to implement the "if function", and is expressed as follows:

Discount $=$ if $\left(\_s t a g e<9 ; 1 ;\right.$ if $\left.\left(\_s t a g e<18 ;\left(1+r^{*}\right) ; 1: 035^{2}\right)\right)$ with _stage representing the cycle number.

\section{Estimating and expressing the cost-effectiveness results-The ICER was} estimated using the Eq. 3. The ICER was initially expressed in U.S. dollars per qualityadjusted progression-free weeks (QAPFW) and then translated into U.S. dollars per qualityadjusted progression-free years (QAPFYs). This translation consisted in multiplying the initial ICER by 52 (total number of weeks in a year).

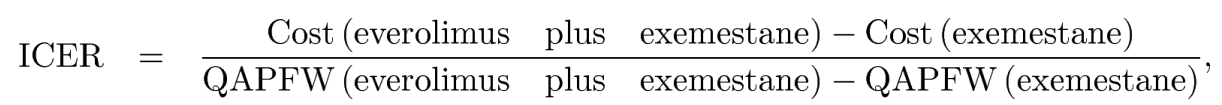

where Cost (everolimus plus exemestane) and Cost (exemestane) were the costs associated with everolimus plus exemestane and exemestane, respectively, and QAPFW (everolimus plus exemestane) and QAPFW (exemestane) represented the quality-adjusted progressionfree weeks gained in both groups.

Sensitivity analyses-Deterministic sensitivity analyses on costs, utilities, and transition probabilities were performed to identify key parameters driving the model (tornado analysis). These analyses consisted in varying each key parameter within the lower and upper bound of its confidence interval. When the confidence interval was not available for the parameter, it was varied within a \pm 25 or $\pm 50 \%$ range [respectively for solid estimates (e.g., Medicare cost estimates) and estimates based on different studies] in relation to its 
base-case scenario value (Table 3). Additionally, the impact of the joint uncertainty around the key parameters of the model on the ICER was assessed through a probabilistic sensitivity analysis (PSA) using a second-order Monte-Carlo simulation (Table 3).

PSA was conducted through the assignment of beta distributions for transition probabilities from the stable state without AEs to the stable state with AEs and gamma distributions for utilities. Disutilities were assigned uniform distributions, while costs were assigned normal distributions, except those related the management of AEs (gamma distribution). The parameters $(\lambda$ and $\gamma$ ) of the time-dependent transition probabilities (from the stable state with or without AEs to progression) were assigned normal distributions. If standard deviation (SD) values of the input parameters for the model were not available, we used the range rule of thumb to obtain rough estimates of SDs. This was achieved by dividing the range of possible values of the parameters by four, assuming that $95 \%$ of sample values lie within two standard deviations of the mean for the majority of datasets. The Monte-Carlo simulation was run for 10,000 iterations to obtain the mean incremental cost per QAPFW and the incremental cost per QAPFY with their respective $95 \%$ confidence intervals. Costeffectiveness acceptability curves (CEAC) were created from these analyses.

\section{Results}

\section{Base-case analysis results}

The results of the base-case analysis showed that the total cost of everolimus/exemestane $(\$ 63,584)$ was 21 times higher than the cost of exemestane alone $(\$ 3,010)$. In terms of effectiveness, the addition of everolimus to exemestane generated 2.3 times more QAPFW (21.24 weeks) than exemestane alone (9.36 weeks). Taken together, the ICER of the comparison of everolimus plus exemestane compared to exemestane was estimated at $\$ 5,105.74 / \mathrm{QAPFW}$. This incremental ratio was translated into \$265,498.5/QAPFY. Based on the WTP threshold of $\$ 149,895 / \mathrm{QAPFY}$, the combination of everolimus plus exemestane appeared to be not cost-effective.

\section{Sensitivity analyses}

Univariate sensitivity analyses showed important variations of the ICER, ranging between $\$ 189,836.4$ and $\$ 530,947 / \mathrm{QAPFY}$. The tornado analysis suggested that the key drivers of our model, by order of importance, were (i) health utility values for stable disease; (ii) acquisition costs of everolimus; and (iii) and transition probabilities from stable to progression states, respectively for treatment arms 0 and 1 (see Fig. 2). That being said, the direction and interpretation of the base-case results remained materially unchanged. In other words, considering a willingness to pay of $\$ 149,895$, the addition of everolimus to exemestane was not found to be cost-effective, even after univariate sensitivity analyses (Tables 4, 5).

The Monte-Carlo simulation showed results that were very similar to the base-case analysis. The mean costs for exemestane alone vs. everolimus/exemestane were $\$ 3,069$ and $\$ 64,838$, respectively. The mean QAPFW was 9.55 and 21.65 weeks for exemestane and everolimus/ exemestane, respectively. The mean incremental cost per QAPFW gained (2.5th percentile; 
97.5th percentile) and mean incremental cost per QAPFY gained (2.5th percentile; 97.5th percentile) for the combination of everolimus plus exemestane compared to exemestane alone were $\$ 5,105(\$ 4,546 ; \$ 6,254)$ and $\$ 265,460$ (\$236,392; $\$ 325,208)$, respectively. The results of the probabilistic sensitivity analyses using Monte-Carlo second-order simulations also suggested that if a QAPFY was worth $\$ 149,895$, then the combination of everolimus and exemestane would have $0 \%$ chance of being cost-effective. These results are illustrated in Fig. 3.

\section{Discussion}

We performed a cost-effectiveness study evaluating everolimus plus exemestane in the setting of hormone receptor positive $\mathrm{mBC}$, utilizing available efficacy data from the BOLERO-2 trial. We built our model utilizing the primary endpoint of the trial, i.e., local PFS assessment, which was what both the FDA and EMA approval were based on. According to the results of our base-case analysis, everolimus/exemestane was not costeffective compared to exemestane alone, provided that the ICER $(\$ 265,498.50)$ was above the WHO defined WTP threshold. Even when utilizing alternate definitions of WTP thresholds, ranging between $\$ 100,000$ and $\$ 200,000$, this combination was still not found to be cost-effective. Our results did not change significantly after conducting univariate sensitivity analyses and Monte-Carlo simulation. Overall, the combination of everolimus plus exemestane was found not to be cost-effective from a Medicare payer perspective.

Recently, the United Kingdom (UK) National Institute for Health and Care Excellence (NICE) conducted an appraisal of everolimus in combination with exemestane based on the evidence provided by the manufacturer (Novartis, UK) [13]. NICE did not recommend the combination for the treatment of women with hormone receptor positive MBC who had progressed or recurred after prior therapy with a non-steroidal aromatase inhibitor. It is worth mentioning three notable differences between our economic model and the one presented by the manufacturer to NICE.

First, the model submitted by the manufacturer utilized PFS data by central assessment, while we utilized locally assessed PFS data. Efficacy of anti-cancer drugs is determined locally in routine clinical setting, thus, it is important for economic assessments to reflect this fact by utilizing locally assessed PFS data. Additionally, using PFS data based on central assessment introduces a greater potential for bias in the economic assessment [14].

Second, our model took improvements in PFS at face value and did not make assumptions regarding the relationship between PFS and OS. The model presented to NICE made the assumption that the central PFS benefit associated with everolimus in BOLERO-2 would translate into an incremental OS benefit of 10.5 months. This estimate was obtained using the product of the hazard ratios for OS, reported in 6 randomized trials comparing endocrine therapy vs. chemotherapy, and the hazard ratio for OS when tamoxifen alone was compared with everolimus plus tamoxifen [14]. Clearly this assumption has recently proven to be rather inaccurate, since final OS results from BOLERO-2 reported a non-significant median OS difference between both arms of only 4.4 months (31 vs. 26.6 months; hazard ratio = 0.89 [95 \% CI 0.73-1.10]; $p=0.1426$ ) [15]. 
The availability of other effective therapeutic options in MBC patients on phase 3 trials could clearly impact and confound any differences in OS between control and experimental arms. For this reason, PFS has been increasingly utilized as a primary study endpoint. Many drugs like everolimus are FDA approved based only on the endpoint PFS, even in the absence of any significant difference in OS. Therefore, PFS should be utilized as an endpoint in economic analyses of systemic therapies in patients with MBC. This approach has the advantage to avoid the need to introduce dubious assumptions on how differences in PFS could potentially affect OS, especially, when a trial like BOLERO-2 fails to demonstrate any significant OS differences. Third, transition probabilities between health states, used to populate our model, were obtained from a survival modeling of the reconstructed IPD of the BOLERO-2 trial [9]. The authors of this study used both graphical and analytical tools to fit appropriate distributions to the IPD from the trial, contrary to the manufacturer of everolimus.

Thirdly, our economic model explicitly accounts for grade 3-4 adverse events associated with the treatment arms of the BOLERO-2 trial, contrary to the model proposed by Novartis. The results obtained from our model are therefore more comprehensive and accurate than those from Novartis.

Our economic assessment is subject to some limitations. First, our analysis was based on initial results from BOLERO-2, prior to the recently presented OS results. Thus, it is possible that any significant difference in OS favoring everolimus would have affected assumptions in our economic model. Another limitation of our model is that it did not explicitly account for dose adjustments (interruptions or reductions) which occurred in treatment arms of the BOLERO-2 trial (63 and $14 \%$, respectively, for the combination and the monotherapy). That being said, dose adjustments were considered in deterministic and probabilistic sensitivity analyses, through the variation of both transition probabilities and acquisition costs (ultimately costs of treatment per cycle) of the treatment arms of the trial. Both analyses did not materially change the base-case results. In the same vein, we made several assumptions in order to populate our economic model, which ultimately did not change the results of our base-case analysis. Fourth, our economic assessment was conducted from the perspective of the public payer, while several guidelines regarding the conduct of cost-effectiveness analysis recommend the use of the societal perspective for comprehensiveness purpose. It is our belief that the use of the societal perspective in this study would have not changed the results of our analysis.

The primary implication of our findings, from a public policy perspective, is that everolimus should not be a preferred drug in the treatment of patients with hormone receptor positive MBC. From a Medicare payer perspective, everolimus should be limited to patients most likely to benefit from the combination of everolimus plus exemestane. Since clinical data suggests a greater likelihood of clinical benefit when the mutation and/or gene signature is present, limiting treatment of everolimus to the subset of $\mathrm{mBC}$ patients with phosphatidylinositol 3' kinase (PIK3CA) could be cost-effective strategy [6, 16]. A more recent study demonstrated favorable response rates in patients who were prescribed everolimus or another mTOR inhibitor based on the presence of mutations in either PIK3CA or downstream AKT [17]. Currently, it is unknown how genomic profiling will impact the 
cost-effectiveness of everolimus in the setting of hormone receptor positive breast cancer. Further economic evaluations are needed to assess the value of everolimus in the treatment of $\mathrm{mBC}$.

In conclusion, our study, based on the interim results of the BOLERO-2 trial, suggests that the combination of everolimus/exemestane is not cost-effective compared to exemestane alone. Our economic assessment is based on a single trial with limited data, thus, further economic evaluations are recommended to assess the cost-effectiveness of everolimus plus exemestane compared to exemestane alone in sub-groups of postmenopausal women with HR positive $\mathrm{mBC}$.

\section{Acknowledgments}

The authors would like to thank Gordon Blackhouse (Programs for Assessment of Technology in Health (PATH) Research Institute, Hamilton, Ontario, Canada), Askal Ali, Dr. Janet Barber, and Dr. Ellen Campbell (Division of Economic, Social and Administrative Pharmacy, College of Pharmacy and Pharmaceutical Sciences, Florida A\&M University (FAMU), Tallahassee, FL, United States) for their insightful comments on earlier versions of the paper.

\section{References}

1. American Cancer Society. Cancer Facts \& Figures 2013. American Cancer Society; Atlanta: 2013.

2. Finn R, Crown J, Boer K, et al. Results of a randomized phase 2 study of PD 0332991, a cyclindependent kinase (CDK) 4/6 inhibitor, in combination with letrozole vs letrozole alone for first-line treatment of ER/HER2-advanced breast cancer (BC). Cancer Res. 2012; 72(24 Suppl):S1-S6.

3. Dees EC, Carey LA. Improving endocrine therapy for breast cancer: it's not that simple. J Clin Oncol. 2013; 31:171-173. [PubMed: 23233714]

4. Zardavas D, Fumagalli D, Loi S. Phosphatidylinositol 3-kinase/AKT/mammalian target of rapamycin pathway inhibition: a breakthrough in the management of luminal (ER/HER2-) breast cancers? Curr Opin Oncol. 2012; 24:623-634. [PubMed: 22960556]

5. Villarreal-Garza C, Cortes J, Andre F, et al. mTOR inhibitors in the management of hormone receptor-positive breast cancer: the latest evidence and future directions. Ann Oncol. 2012; 23:2526-2535. [PubMed: 22553196]

6. Baselga J, Semiglazov V, van Dam P, et al. Phase II ran- domized study of neoadjuvant everolimus plus letrozole compared with placebo plus letrozole in patients with estrogen receptor-positive breast cancer. J Clin Oncol. 2009; 27:2630-2637. [PubMed: 19380449]

7. Baselga J, Campone M, Piccart M, et al. Everolimus in postmenopausal hormone-receptor-positive advanced breast cancer. N Engl J Med. 2012; 366:520-529. [PubMed: 22149876]

8. Piccart M, Baselga J, Noguchi S, et al. Final progression- free survival analysis of BOLERO-2: a phase III trial of everolimus for postmenopausal women with advanced breast cancer. Chemotherapy. 2012; 42:59.

9. Diaby V, Adunlin G, Montero AJ. Survival modeling for the estimation of transition probabilities in model-based economic evaluations in the absence of individual patient data: a tutorial. Pharmacoeconomics. 2013; 32:101-108. [PubMed: 24338265]

10. Liou S, Stephens J, Carpiuc K, et al. Economic burden of haematological adverse effects in cancer patients. Clin Drug Invest. 2007; 27:381-396.

11. Evans DB, Hurley SF. The application of economic evaluation techniques in the health sector: the state of the art. J Int Dev. 1995; 7:503-524.

12. Gold, MR. Cost-effectiveness in health and medicine. Oxford University Press; New York: 1996.

13. The National Institute for Health and Care Excellence (NICE). Everolimus in combination with exemestane for treating advanced HER2-negative hormone-receptor-positive breast cancer after endocrine therapy. 2013. 
14. Burke MJ, Garrett Z, George E, et al. NICE guidance on everolimus in combination with exemestane for treatment of advanced HER2-negative, hormone-receptor-positive breast cancer after endocrine therapy. Lancet Oncol. 2013; 14:1049-1050.

15. Piccart, M.; Hortobagyi, GN.; Campone, M., et al. Everolimus plus exemestane for hormone receptor-positive $(\mathrm{HR}+)$, human epidermal growth factor receptor-2-negative (HER2-) advanced breast cancer (BC): overall survival results from BOLERO-2; Oral Presentation Abstract \#LBA1. European Breast Cancer Conference (EBCC-9); Glasgow, Scotland. 2014. 2014.

16. Loi S, Michiels S, Baselga J, et al. PIK3CA genotype and a PIK3CA mutation-related gene signature and response to everolimus and letrozole in estrogen receptor positive breast cancer. PLoS One. 2013; 8:e53292. [PubMed: 23301057]

17. André F, Bachelot T, Commo F, et al. Comparative genomic hybridisation array and DNA sequencing to direct treatment of metastatic breast cancer: a multicentre, prospective trial (SAFIR01/UNICANCER). Lancet Oncol. 2014; 15:267-274. [PubMed: 24508104]

18. Centers for Medicare and Medicaid Services. Medicare physician fee schedule (MPFS). 2014.

19. Centers for Medicare and Medicaid Services. Payment allowance limits for medicare. 2013 Part B. Drugs.

20. Xie J, Diener M, De G, et al. Budget impact analysis of everolimus for the treatment of hormone receptor positive, human epidermal growth factor receptor-2 negative (HER2-) advanced breast cancer in the United States. J Med Econ. 2012; 16:278-288. [PubMed: 23153318]

21. Pfuntner, A.; Wier, LM.; Steiner, C. Costs for hospital stays in the United States, 2011. Agency for Health Care Policy and Research (US); 2013.

22. Lloyd A, Nafees B, Narewska J, et al. Health state utilities for metastatic breast cancer. Br J Cancer. 2006; 95:683-690. [PubMed: 16967055] 


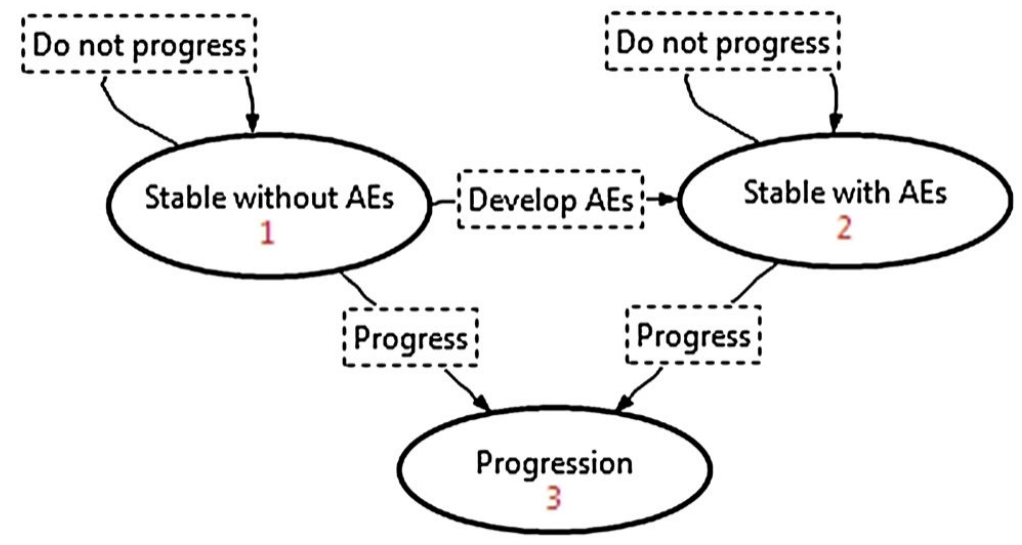

Fig. 1.

Markov state transition diagram. The Markov model simulated two hypothetical cohorts of postmenopausal women with advanced breast cancer, one treated with everolimus plus exemestane and the other exemestane alone. The model considered three health states: stable disease with no adverse events, stable disease with adverse events, and disease progression. Patients would exit the model once they enter the disease progression state. AEs adverse events 


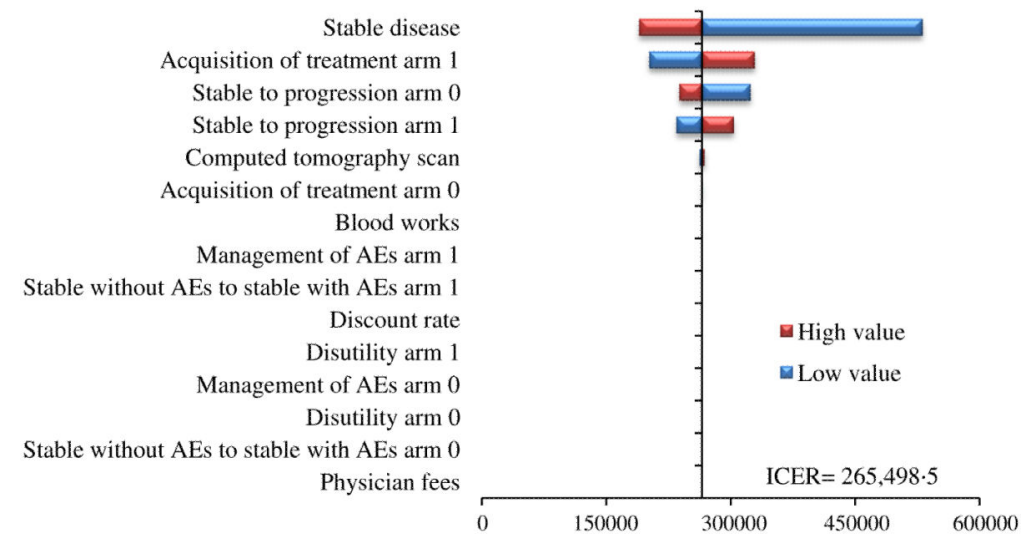

Fig. 2.

Tornado diagram. AEs adverse events, ICER incremental cost-effectiveness ratio 


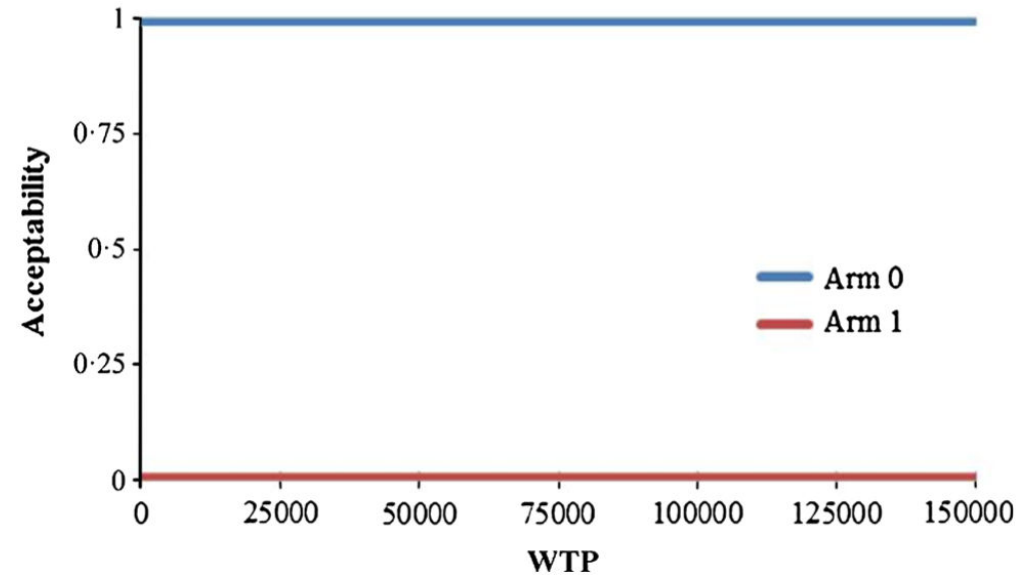

Fig. 3.

Cost-effectiveness acceptability curves. WTP willingness to pay, Arm 0 exemestane, Arm 1 everolimus + exemestane 


\section{Table 1}

Medical resource use and costs

\begin{tabular}{|c|c|c|c|c|}
\hline Resource & Unit & $\begin{array}{l}\text { Cost in } 2013 \\
\text { US } \$\end{array}$ & Assumptions & Reference \\
\hline \multicolumn{5}{|l|}{ Medical visit } \\
\hline Physician fees & 1 consultation & 108.53 & $\begin{array}{l}100 \% \text { of patients covered by } \\
\text { Medicare }\end{array}$ & {$[18]$} \\
\hline \multicolumn{5}{|l|}{ Acquisition of treatment } \\
\hline Arm 1 & Every 6 weeks & $11,733.12$ & $\begin{array}{l}\text { Everolimus } 10 \mathrm{mg} \text { daily dose } \\
\quad \$ 273.36) \text {, or a weekly cost of } \\
\$ 1,913.52 \text {. Exemestane } \\
25 \mathrm{mg} \text { at a weekly cost of } \\
\$ 42\end{array}$ & {$[19]$} \\
\hline Arm 0 & Every 6 weeks & 252 & $\begin{array}{l}\text { Exemestane } 25 \mathrm{mg} \text { daily } \\
(\$ 6.00) \text {, or a weekly cost of } \\
\$ 42\end{array}$ & {$[20]$} \\
\hline \multicolumn{5}{|c|}{ Management of adverse events (grade 3/4) } \\
\hline Arm 1 & 1 time & $3,161.5435$ & $\begin{array}{l}\text { Weighted average of } \\
\text { components of interest }\end{array}$ & {$[7,10,21]$} \\
\hline Arm 0 & 1 time & 253.784791 & $\begin{array}{l}\text { Weighted average of } \\
\text { components of interest }\end{array}$ & {$[7,10,21]$} \\
\hline $\begin{array}{l}\text { Computed tomography } \\
\text { (CT) scan }\end{array}$ & $\begin{array}{l}1 \text { scan every } \\
6 \text { weeks }\end{array}$ & 1037.65 & $\begin{array}{l}\text { Assume scan repeated every } \\
6 \text { weeks as per BOLERO-2 }\end{array}$ & {$[18]$} \\
\hline \multicolumn{5}{|l|}{ Laboratory tests } \\
\hline Blood work & Every 6 weeks & 65.58 & 32.79 every 3 weeks & [18] \\
\hline
\end{tabular}

* Original costs inflated using Consumer Price Index (CPI) inflation calculator from the Bureau Labor of Statistics, available at http://www.bls.gov/ data/inflation_calculator.htm 


\section{Table 2}

Utility and disutility weights

\begin{tabular}{llll}
\hline Utilities & $\begin{array}{l}\text { Weighted } \\
\text { average } \\
\text { values }\end{array}$ & $\begin{array}{l}\text { Original } \\
\text { values } \\
\text { (distributions) }\end{array}$ & Source \\
\hline $\begin{array}{l}\text { Base state-stable disease } \\
\text { Utility decrement }\end{array}$ & 0.715 & 0.715 & {$[22]$} \\
Grade 3 and 4 adverse events & & & \\
Arm 1 & 0.01208 & $0.151(8 \%)$ & {$[7,22]$} \\
Stomatitis & 0.00906 & $0.151(6 \%)^{a}$ & {$[7,22]$} \\
Anemia & 0.00604 & $0.151(4 \%)^{a}$ & {$[7,22]$} \\
Dyspnea & 0.00604 & $0.151(4 \%)^{a}$ & {$[7,22]$} \\
Hyperglycemia & 0.0046 & $0.115(4 \%)$ & {$[7,22]$} \\
Fatigue & 0.00453 & $0.151(3 \%)^{a}$ & {$[7,22]$} \\
Pneumonitis & & & \\
Arm 0 & 0.00151 & $0.151(1 \%)$ & {$[7,22]$} \\
Stomatitis & 0.00151 & $0.151(1 \%)^{a}$ & {$[7,22]$} \\
Anemia & 0.00151 & $0.151(1 \%)^{a}$ & {$[7,22]$} \\
Dyspnea & 0.00151 & $0.151(1 \%)^{a}$ & {$[7,22]$} \\
Hyperglycemia & 0.00115 & $0.115(1 \%)$ & {$[7,22]$} \\
Fatigue & 0 & $0.151(0 \%)^{a}$ & {$[7,22]$} \\
Pneumonitis & & & \\
\hline
\end{tabular}

Treatment arm 0: exemestane only; treatment arm 1: everolimus + exemestane

${ }^{a}$ To obtain the utility decrements for adverse events, for which values were not available in the literature, we multiplied the highest utility decrement value available $(0.151)$ by the proportion of patients experiencing these adverse events 
Table 3

Parameter values and ranges examined in sensitivity analyses

\begin{tabular}{|c|c|c|c|c|}
\hline \multirow[t]{2}{*}{ Variable } & \multirow[t]{2}{*}{$\begin{array}{l}\text { Base-case } \\
\text { value }\end{array}$} & \multicolumn{2}{|c|}{$\begin{array}{l}\begin{array}{l}\text { Range of uncertainty used for } \\
\text { one-way deterministic sensitivity } \\
\text { analysis }\end{array} \\
\end{array}$} & \multirow[t]{2}{*}{$\begin{array}{l}\text { Distributions used for probabilistic } \\
\text { sensitivity analysis }\end{array}$} \\
\hline & & Low value & High value & \\
\hline \multicolumn{5}{|l|}{ Probability parameters } \\
\hline \multicolumn{5}{|l|}{ Arm 0} \\
\hline $\begin{array}{l}\text { Stable (with or without AEs) to } \\
\text { progression }\end{array}$ & $\begin{array}{l}\text { Eq. } 1 \text { (Log- } \\
\text { logistic) }\end{array}$ & $\begin{array}{l}-25 \% \times \\
\text { Eq. } 1\end{array}$ & $\begin{array}{l}+25 \% \times \\
\text { Eq. } 1\end{array}$ & $\begin{array}{l}\text { Normal distribution for the parameters of } \\
\text { the Log-logistic equation }\left(\lambda_{0} ; \gamma_{0}\right)\end{array}$ \\
\hline Stable without AEs to stable with AEs & 0.0005 & 0.00038 & 0.00063 & Beta \\
\hline \multicolumn{5}{|l|}{ Arm 1} \\
\hline $\begin{array}{l}\text { Stable (with or without AEs) to } \\
\text { progression }\end{array}$ & $\begin{array}{l}\text { Eq. } 2(\text { Log- } \\
\text { logistic) }\end{array}$ & $\begin{array}{l}-25 \% \times \\
\text { Eq. } 2\end{array}$ & $\begin{array}{l}+25 \% \times \\
\text { Eq. } 2\end{array}$ & $\begin{array}{l}\text { Normal distribution for the parameters of } \\
\text { the Log-logistic equation }\left(\lambda_{1} ; \gamma_{1}\right)\end{array}$ \\
\hline Stable without AEs to stable with AEs & 0.006 & 0.0045 & 0.0075 & Beta \\
\hline \multicolumn{5}{|l|}{ Costs (2013 U.S. dollars) } \\
\hline Physician fees & 108.53 & 81.3975 & 135.6625 & Normal \\
\hline \multicolumn{5}{|l|}{ Acquisition of treatment } \\
\hline Arm 1 & $11,733.12$ & $8,799.84$ & $14,666.4$ & Normal \\
\hline Arm 0 & 252 & 189 & 315 & Normal \\
\hline \multicolumn{5}{|l|}{ Management of adverse events } \\
\hline Arm 1 & $3,161.5435$ & $1,580.77175$ & $4,742.31525$ & Gamma \\
\hline Arm 0 & 253.784791 & 126.8923955 & 380.6771865 & Gamma \\
\hline Computed tomography scan & $1,037.65$ & 778.2375 & $1,297.0625$ & Normal \\
\hline \multicolumn{5}{|l|}{ Laboratory tests } \\
\hline Blood works & 65.58 & 49.185 & 81.975 & Normal \\
\hline \multicolumn{5}{|l|}{ Utility weights } \\
\hline ...Stable disease & 0.715 & 0.3575 & 1 & Gamma \\
\hline \multicolumn{5}{|l|}{ Disutility (Grade $3 / 4$ adverse events) } \\
\hline \multicolumn{5}{|l|}{ Arm 1} \\
\hline Stomatitis & 0.01208 & - & - & - \\
\hline Anemia & 0.00906 & - & - & - \\
\hline Dyspnea & 0.00604 & - & - & - \\
\hline Hyperglycemia & 0.00604 & - & - & - \\
\hline Fatigue & 0.0046 & - & - & - \\
\hline Pneumonitis & 0.00453 & - & - & - \\
\hline Total disutility & 0.04235 & 0.021175 & 0.063525 & Uniform \\
\hline \multicolumn{5}{|l|}{ Arm 0} \\
\hline Stomatitis & 0.00151 & - & - & - \\
\hline Anemia & 0.00151 & - & - & - \\
\hline Dyspnea & 0.00151 & - & - & - \\
\hline Hyperglycemia & 0.00151 & - & - & - \\
\hline Fatigue & 0.00115 & - & - & - \\
\hline Pneumonitis & 0 & - & - & - \\
\hline
\end{tabular}




\begin{tabular}{|c|c|c|c|c|}
\hline \multirow[t]{2}{*}{ Variable } & \multirow[t]{2}{*}{$\begin{array}{l}\text { Base-case } \\
\text { value }\end{array}$} & \multicolumn{2}{|c|}{$\begin{array}{l}\text { Range of uncertainty used for } \\
\text { one-way deterministic sensitivity } \\
\text { analysis }\end{array}$} & \multirow[t]{2}{*}{$\begin{array}{l}\text { Distributions used for probabilistic } \\
\text { sensitivity analysis }\end{array}$} \\
\hline & & Low value & High value & \\
\hline Total disutility & 0.00719 & 0.003595 & 0.010785 & Uniform \\
\hline Discount rate & $3.5 \%$ & $0 \%$ & $5 \%$ & - \\
\hline
\end{tabular}


Table 4

Cost-effectiveness analysis results

\begin{tabular}{lllllll}
\hline & Cost $\mathbf{\$}$ ) & $\begin{array}{l}\text { Incremental } \\
\text { cost }(\$)\end{array}$ & $\begin{array}{l}\text { Effectiveness } \\
(\mathbf{Q A P F W})\end{array}$ & $\begin{array}{l}\text { Incremental } \\
\text { effectiveness (QAPFW) }\end{array}$ & $\begin{array}{l}\text { ICER } \\
(\mathbf{\$ Q A P F W})\end{array}$ & $\begin{array}{l}\text { ICER } \\
(\mathbf{\$ Q A P F Y})\end{array}$ \\
\hline Everolimus + exemestane & $63,583.52$ & $60,573.16$ & 21.24 & 11.88 & $5,105.74$ & $265,498.5$ \\
Exemestane & $3,010.36$ & - & 9.36 & - & - & \\
\hline
\end{tabular}

$I C E R$ incremental cost-effectiveness ratio, $Q A P F W$ quality-adjusted progression-free weeks, $Q A P F Y$ quality-adjusted progression-free years, $\$$ dollars 


\section{Table 5}

One-way sensitivity analysis results

\begin{tabular}{|c|c|c|}
\hline \multirow[t]{2}{*}{ Variable } & \multicolumn{2}{|c|}{$\underline{\text { ICER (\$/QAPFY) }}$} \\
\hline & Low value & High value \\
\hline \multicolumn{3}{|l|}{ Probability parameters } \\
\hline \multicolumn{3}{|l|}{ Arm 0} \\
\hline $\begin{array}{l}\text { Stable (with or without AEs) to } \\
\text { progression }\end{array}$ & $323,926.72$ & $238,211.48$ \\
\hline Stable without AEs to stable with AEs & $265,498.48$ & $265,497.96$ \\
\hline \multicolumn{3}{|l|}{ Arm 1} \\
\hline $\begin{array}{l}\text { Stable (with or without AEs) to } \\
\text { progression }\end{array}$ & $234,651.56$ & $303,709.12$ \\
\hline Stable without AEs to stable with AEs & $265,415.28$ & $265,580.12$ \\
\hline \multicolumn{3}{|l|}{ Costs (2013 U.S. dollars) } \\
\hline Physician fees & $265,497.96$ & $265,497.96$ \\
\hline \multicolumn{3}{|l|}{ Acquisition of treatment } \\
\hline Arm 1 & $201,956.04$ & $329,040.4$ \\
\hline Arm 0 & $266,099.6$ & $264,896.84$ \\
\hline \multicolumn{3}{|l|}{ Management of adverse events } \\
\hline Arm 1 & $265,316.48$ & $265,679.96$ \\
\hline Arm 0 & $265,498.48$ & $265,497.96$ \\
\hline Computed tomography scan & $262,354.04$ & $268,642.4$ \\
\hline \multicolumn{3}{|l|}{ Laboratory tests } \\
\hline Blood works & $265,299.32$ & $265,697.12$ \\
\hline \multicolumn{3}{|l|}{ Utility } \\
\hline Stable disease & $530,947.04$ & $189,836.4$ \\
\hline \multicolumn{3}{|l|}{ Disutility (Grade 3/4 AEs) } \\
\hline Arm 1 & $265,510.44$ & 265,486 \\
\hline Arm 0 & $265,497.96$ & $265,498.48$ \\
\hline Discount rate & $265,452.72$ & $265,517.2$ \\
\hline
\end{tabular}

ICERs incremental cost-effectiveness ratios, AEs adverse events, $\$$ dollars, $Q A P F Y$ quality-adjusted life years 